Vigiliae Christianae 35, 24-31; North-Holland Publishing Company 1981

\title{
DER MENSCH ALS POLITISCHES LEBEWESEN BEI BASILIOS DEM GROSSEN*
}

\author{
VON
}

\author{
TH. NIKOLAOU
}

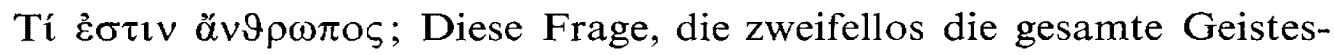
geschichte durchzieht, zielt auf das „Wesen des Menschen“ ab, d.h. auf etwas, das nicht geradewegs zum Gegenstand wissenschaftlicher Prüfung und Eruierung werden kann. Die Vielfalt der Antworten auf diese Frage (vom aristotelischen animal rationale bis zum schellerschen ,das lebendige $\mathrm{x}$, das Gott sucht", vom klassischen homo sapiens bis zum homo insipiens von Ortega y Gasset, vom homo faber bis zum marxistischen Konzept des Menschen als „ensemble der gesellschaftlichen Verhältnisse“) ist daher nur allzu verständlich.

Eine solche Antwort stellt auch das bekannte aristotelische Schlagwort dar, das den Menschen als ,von Natur aus politisches Lebewesen“"

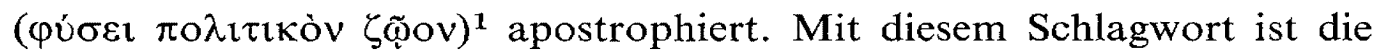
nacharistotelische Denkgeschichte durchaus vertraut. ${ }^{2}$ Auch dem griechisch geschulten christlichen Denker, Basilios dem Großen ist die Bezeichnung des Menschen als „politisches Lebewesen“ bekannt. Wie ist sie aber bei ihm zu verstehen? Zur Klärung dieser Frage möchte ich erstens die betreffende Stelle bei Basilios besprechen und zweitens den dadurch herauszuschälenden Sinn des Ausdruckes mit dem aristotelischen Kontext und den Gegebenheiten der griechischen Philosophie überhaupt vergleichen.

1. Die Bezeichnung des Menschen als „politisches Lebewesen“ begegnet uns in der ersten der beiden Homilien von Basilios zum 14. Psalm (6: PG, 29,261C). Der Inhalt dieser Homilie, wie auch der übrigen Psalmenhomilien, dient erbaulichen und nicht exegetischen Zwecken. Dies geht auch aus der betreffenden Stelle hervor, an der der Homilet sich auf Vers 5 des Psalms ("der sein Geld nicht mit Zinsen verleiht") ${ }^{3}$ nur inhaltlich bezieht und anstelle dieses Verses Mt 5,42 (,Dem, der dich bittet, gib, und wer bei dir borgen will, von dem wende dich nicht $a b^{\text {c) }}$ ) zum Ausgangspunkt seiner Überlegungen macht.

$0042-6037 / 1981 / 0000-0000 / \$ 2.50$ (C) North-Holland Publishing Company 


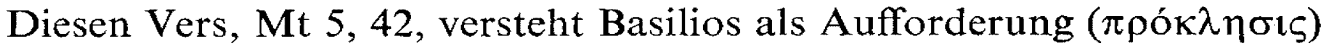

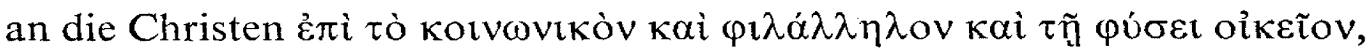
d.h. zur Gemeinschaft, Nächstenliebe und zur natürlichen Verwandschaft

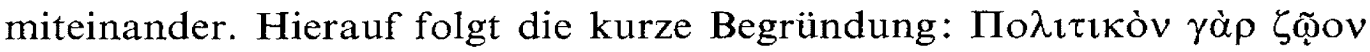

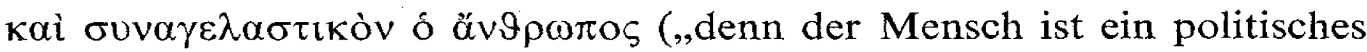
und in Scharen zusammenlebendes Wesen“). Und er fährt fort: „In der

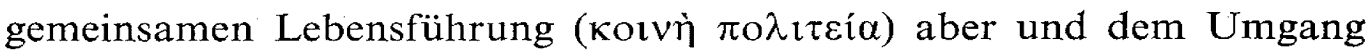

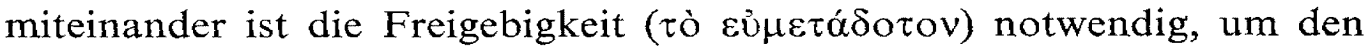
Notleidenden aufzuhelfen. ,Dem, der dich bittet, gib'. Es (sc. dieses Wort) will, daß du denjenigen gegenüber, die dich bitten, einerseits aus Liebe einfach offen bist, andererseits aber daß du die Dürftigkeit eines jeden, der dich bittet, durch die Vernunft unterscheidest".

Worum es hier in erster Linie geht, ist sicherlich nicht ein theoretischer Exkurs über das Wesen des Menschen, sondern die konkrete Ausgestaltung des Lebens der christlichen Gemeinschaft gemäß dem Evangelium. Mit $\pi \circ \lambda \imath \tau \varepsilon i \alpha$ ist hier nicht eine politische Ordnung bzw. eine Staatsform im klassischen Sinne des Wortes gemeint, d.h. der gut organisierten Gemeinschaft, die von Gesetzen durchwaltet wird und konkrete Ziele verfolgt ; ${ }^{4}$ auch jene andere klassische Bedeutung des Terminus, nach der

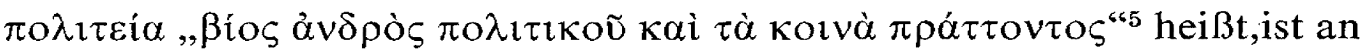
dieser Stelle nicht beinhaltet. Der Ausdruck weist bei den Kirchenvätern bekanntlich oft die Bedeutung entweder allgemein der Lebensweise (way of life - ohne ethische Wertung) oder des tugendhaften, vollkommenen, evangeliumsgemäßen Lebens auf. ${ }^{6}$ Basilios verwendet wiederholt den Be-

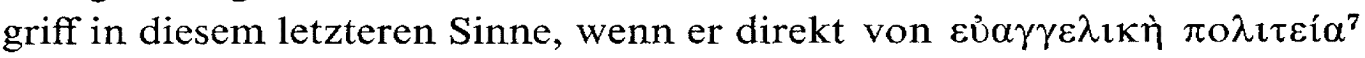

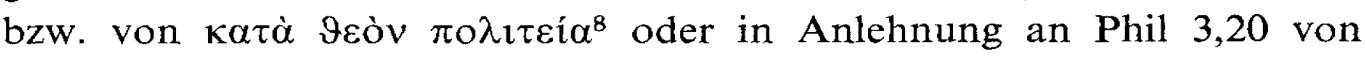
$\pi \circ \lambda i ́ \tau \varepsilon v \mu \alpha \dot{\varepsilon} \nu$ oủ $\rho \alpha v o \tilde{\varsigma} \varsigma^{9}$ spricht. Hierbei sind $\pi \circ \lambda \iota \tau \varepsilon i \alpha$ und $\pi \circ \lambda i \tau \varepsilon v \mu \alpha$ gleichzusetzen. Die vollkommene Lebensführung ist für den Christen in der kirchlichen Gemeinschaft gewährleistet. Hierin ist wohl auch der Grund zu' sehen, warum Basilios sich so sehr für das organisierte Koino-

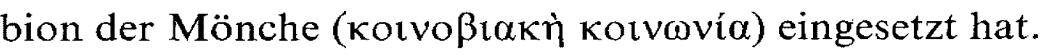

Die Kirche, insbesondere die konkrete kirchliche Gemeinde, ist die - Polis der Christen. Es ist daher nicht zufällig, wenn Basilios in dem Begriff $\pi$ ó $\lambda \iota \varsigma$ von Psalm 59,11 die Kirche vermutet; die Kirche ist eine „Polis, weil sie eine Versammlung ist, die rechtmäßig verwaltet wird“ ( $\sigma v ́ \sigma \tau \eta \mu \alpha$

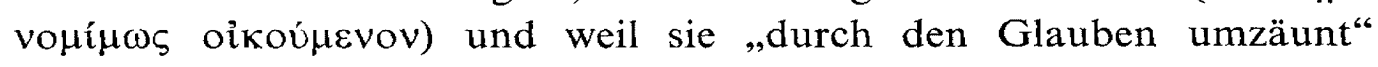
ist. ${ }^{10}$

Die Kirche, betont er nachdrücklich an einer anderen Stelle, ${ }^{11}$ ist die

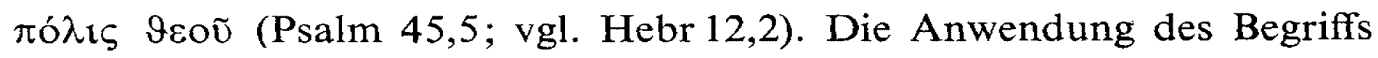


geschieht hier nicht unbedacht. Er zitiert die überlieferte stoische Defini-

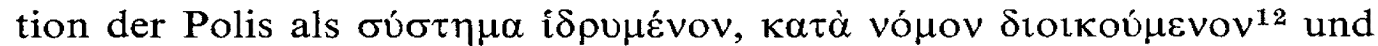
findet diese Definition passend für das "himmlische Jerusalem“ und die „himmlische Polis“; denn diese Polis ist auch eine „geordnete Versammlung $(\sigma u ́ \sigma \tau \eta \mu \alpha)$ der Erstgeborenen, die im Himmel aufgezeichnet sind“ (Hebr 12,23). Die Polis Gottes beschränkt sich allerdings nicht auf das

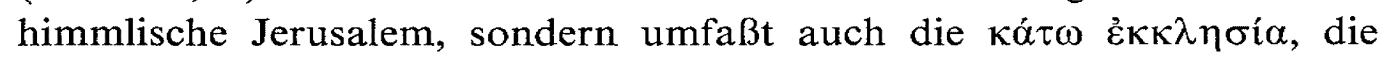
kirchliche Gemeinschaft auf Erden; „die gesamte geistige Schöpfung von den überirdischen Kräften bis zu den menschlichen Seelen muß man als eine Polis verstehen, die durch die Herrschaft des H1. Geistes erquickt wird“. In der Mitte der kirchlichen Gemeinschaft auf Erden ,hat der Höchste seine Wohnung geheiligt“ (Psalm 45,5). Er herrscht in ihr durch seine Güte, seine Vorsehung und seine Gerechtigkeit. Dadurch erweist sich die Kirche als eine wohlgeordenete Versammlung, als die neue Polis. Jeder Mensch hat sich zu dieser Polis, der kirchlichen Gemeinschaft, hinzuzugesellen. Damit macht man sich zum Soldaten und Athleten Christi, der seine Lebensführung im Himmel hat (Phil 3,20). ${ }^{13}$ Der gerechte Mensch gehört noch nicht ganz zu der Polis des lebendigen Gottes; seine endgültige Einbürgerung ( $\pi \circ \lambda \imath \tau o \gamma \rho \alpha ́ \varphi \eta \sigma \imath \varsigma$ ) erfolgt dann, wenn er die eschatologische Geltung vor Gott erreicht hat. ${ }^{\mathbf{1 4}}$

Kehren wir aber nun zu unserer Stelle (Homilia in psalm. 14,5) zurück. Die $\pi \circ \lambda \imath \tau \varepsilon i ́ \alpha$, um die es sich hier handelt, ist zunächst einfach die Lebens-

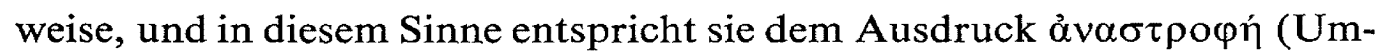
gang, Verkehr) im selben Satz. Beide Begriffe werden jedoch durch die

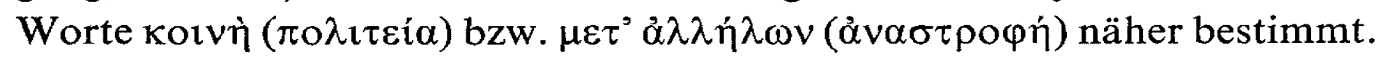
Es geht deshalb nicht um eine beliebige Lebensführung, sondern um die gemeinsame und gemeinschaftliche. Die gemeinsame Lebensführung dient nach Basilios an dieser Stelle dem gesellschaftlichen, sozialen Gleichgewicht. Das Gebot des Herrn, dem zu geben, der dürftig ist und bittet, ist eine Grundvoraussetzung einer wohlgeordneten kirchlichen Gemeinschaft. Das Motiv für die Freigebigkeit erblickt unser Autor in der Liebe. Obwohl er unmittelbar danach auf den „Kommunismus“ der Urgemeinde hinweist, hat er ein konkreteres Ideal vor Augen: die Behebung der Not in der Gemeinschaft. Er setzt sich nicht mit der Leidenschaft eines Johannes Chrysostomos für die Verteilung aller Güter ein, sondern verlangt von seinen Hörern, den Notleidenden behilflich zu werden, ihren Reichtum richtig zu gebrauchen. Nicht Abschaffung des Eigentums steht im Vordergrund, sondern tätige Nächstenliebe. Die Dürftigkeit des Nächsten bestimmt das Maß unserer Freigebigkeit; diese Dürftigkeit 
richtig abzuwägen und ihr entsprechend nachzukommen, ist die Aufgabe der Unterscheidungskraft unserer Vernunft.

Für diese freigebige Haltung des Menschen in der kirchlichen Gemeinschaft sieht Basilios einen gewichtigen Beweggrund auch in seiner natürlichen Veranlagung zum gemeinschaftlichen Leben. Wenn er nun auf das aristotelische Schlagwort über den Menschen als politisches Lebewesen zurückgreift, spricht er eine opinio communis aus, deren Richtigkeit vorausgesetzt wird. Es ist eine schlicht selbstverständliche Aussage, daß der Mensch ein Gemeinschaftswesen und als solches auf seinen Mitmenschen ausgerichtet ist. Diese tiefe Überzeugung bringt der große Kirchenvater zum Ausdruck, wenn er an einer anderen Stelle ${ }^{15}$ fragt: „Wer weiß nicht, daß der Mensch ein zahmes und gemeinschaftliches ( vikóv) und nicht ein alleinlebendes und auch nicht wildes Lebewesen ist

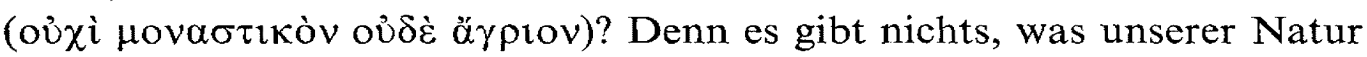
so eigen ist, als das Miteinander-Gemeinschaft-stiften und Einander-bedürfen und das Einander-lieben". Hier wird diese Grundeigenschaft des Menschen deutlicher mit der Liebe in Verbindung gebracht. Das Gebot der Nächstenliebe wäre unerfüllbar, wenn der Mensch als Geschöpf Gottes die entsprechende Kraft nicht von Gott eingegeben bekommen hätte. Diese somit der Natur des Menschen eigene „Liebeskraft“ macht ihn zu einem Lebewesen, das sich zur menschlichen Gemeinschaft hingezogen fühlt und nur in ihr existieren kann. Die hiermit angesprochene Gemeinschaft ist die kirchliche Gemeinschaft, in deren Mitte Gott ist. Die Bestimmung des Menschen als politisches Lebewesen erklärt daher nach Basilios nicht nur den natürlichen Drang zum gemeinschaftlichen Leben, sondern auch die Zweckursache dieses Lebens, Gott im Gebet zu begegnen, Gottes Gnade zu erfahren. Dies ist m.E. der Grund, warum Basilios im weiteren Kontext der betreffenden Homilie mit dem Zeugnis der Schrift Mt 25,35 („Ich war hungrig und ihr habt mir zu essen gegeben“), und Sprüche 19,17 (,Wer sich des Armen erbarmt, leiht an Gott aus") argumentiert. Die Behebung der sozialen Not in der Gemeinschaft stellt selbstverständlich etwas Wertvolles für sich dar; doch darüber hinaus führt sie zu Gott, denn das Unterpfand der Hilfeleistung und der Gabe an

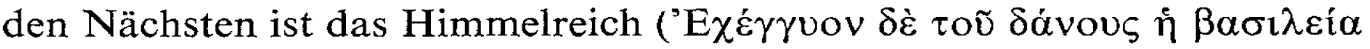
$\tau \tilde{\omega} \nu$ oủ $\alpha \nu \tilde{\omega} \nu)$. Soviel über das Verständnis des Menschen als politisches Lebewesen bei Basilios. Wie verhält sich nun dieses Verständnis zum aristotelischen Kontext und den Gegebenheiten der griechischen Philosophie und seiner Zeit im allgemeinen? 
2. Ein solcher Vergleich führt zu einigen Feststellungen. Die wichtigsten davon möchte ich im Folgenden anführen:

a) Nur äußerlich gesehen könnte man meinen, daß Basilios sich durch die Zitation des aristotelischen Ausdruckes als ein Zeuge des politischen Denkens erweist, welches zu den wesentlichen Kennzeichen der griechischen Philosophie fast seit ihren Anfängen bis zum letzten Philosophen in Byzanz, Georgios Gemistos Plethon, gehört. Daß Basilios sich nicht darin einreihen läßt, erklärt sich nicht durch den Wandel der politischen Strukturen von der griechischen Polis über die hellenistischen Staaten zu dem römischen Imperium. Vielmehr ist dies auf die Tatsache zurückzuführen, daß er die Bezeichnung des Menschen als politisches Wesen im Grunde apolitisch, nämlich nicht in Bezug auf die weltliche Ordnung, verwendet. Der Bereich, in dem der Mensch nach Basilios seine Eigenschaft als Gemeinschaftswesen entfaltet, ist die Kirche, die Polis Gottes. Basilios denkt nicht in den augustinischen Kategorien einer Gegenüberstellung des „göttlichen“ und des ,weltlichen“ Staates. Ihn interessiert primär der Gottesstaat in der konkreten Gestalt der kirchlichen Gemeinde. Der Mensch, der dieser Gemeinschaft angehört, erlangt darin sein Heil und somit seine endgültige Einbürgerung in den Gottesstaat im Eschaton. Der konkreten kirchlichen Gemeinschaft steht der weltliche Staat nicht schlechthin gegenüber. Deshalb ist den weltlichen Herrschern gegenüber Gehorsam angebracht. Es ist kein uneingeschränkter Gehorsam; der Christ hat sich nur jenen Herrschern zu unterwerfen, „bei denen das Gebot Gottes nicht verhindert wird “. ${ }^{16}$ Hierin folgt Basilios der Schrift und meint mit Paulus (Röm 13,1), daß die weltliche Gewalt von Gott herrührt. ${ }^{17}$ Es ist nun die Zeit nach Konstantin dem Großen, in der „das Verhältnis zwischen den beiden endgültigen Mächten der Geschichte sich aus einem feindlichen in ein solches der Zusammenarbeit verwandeln konnte“. ${ }^{18}$ Dieser Verwandlung trägt Basilios hier Rechnung.

b) Die natürliche Eigenschaft des Menschen als Gemeinschaftswesen

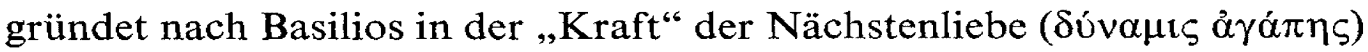
die im Menschen als Geschöpf Gottes keimhaft existiert ( $\sigma \pi \varepsilon \rho \mu \alpha \tau \iota \kappa \tilde{\omega} \varsigma$

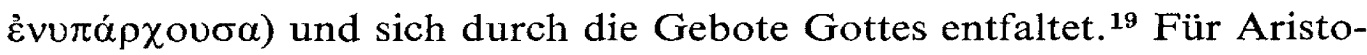
teles ist der Mensch ein politisches Lebewesen, weil er Vernunft ( $\lambda$ ó $\gamma \circ \varsigma$ ) besitzt; darin unterscheidet sich der Mensch von allen anderen Lebewesen. ${ }^{20}$ Zwar haftet die Kraft der Liebe für Basilios der menschlichen Seele an, aber sie läßt sich trotzdem schwerlich mit dem aristotelischen Logos vergleichen.

c) Auch die Ziele, die der Mensch jeweils in seinem Handeln als Gemein- 
schaftswesen verfolgt, lassen sich nicht ohne weiteres vergleichen. $\mathrm{Zu}-$ nächst ist festzustellen, daß sowohl bei Aristoteles als auch bei Basilios durch die Bezeichnung des Menschen als politisches Lebewesen der Bereich der Ethik der Gemeinschaft angesprochen ist. Für Aristoteles vollendete sich die Ethik des Einzelnen, aber auch der Gemeinschaft, in der Polis. In der Ethik der Gemeinschaft, der Politik, sieht er die „Großorganisation der Sittlichkeit" ${ }^{21}$ Eine Antinomie zwischen Politik und Sittlichkeit gab es für ihn nicht. Für Basilios ist dagegen die Sittlichkeit nicht die Schöpfung bloß der menschlichen Gemeinschaft, sondern die Frucht der evangeliumsgemäßen Lebensführung, die sich jedoch in der kirchlichen Gemeinschaft verwirklichen läßt. Dort - bei Aristoteles herrscht das politische Gesetz, das Sittlichkeit ermöglicht und herbeiführt; hier dagegen - in der Kirche - die Gebote Gottes, die über die Sittlichkeit hinausführen. Nicht die Autarkie der Güter und die Eudämonie der Gemeinschaft dient als Ziel in der kirchlichen Gemeinschaft, sondern die Begegnung mit den Mitmenschen. Sie geschieht in der Betätigung der Nächstenliebe, die im konkreten Fall Behebung des Notleidens bedeutet. Aber darüber hinaus - und dies ist das höchste Ziel - bedeutet sie Aneignung der göttlichen Gaben und des Heils, Begegnung mit Gott.

d) Aufgrund der bisherigen Erörterungen läßt sich zum Schluß noch eine Bemerkung machen. Es geht um die vieldiskutierte Frage des Verhältnisses der Kirchenväter zur griechischen Philosophie. Es bleibt unbestreitbar, daß die Verwendung der philosophischen Terminologie bei den Kirchenvätern keine Schwierigkeit bereitet. Ebenso kann man nicht leugnen, da 3 diese Terminologie oft mit gewissen Vorstellungen und Gedanken verknüpft ist. Die Übernahme von philosophischen Begriffen schließt auch die Übernahme entsprechender Gedanken ein, soweit sie nicht der christlichen Offenbarung widersprechen. Da aber durch die christliche Offenbarung zugleich ein anderes Gedankensystem mitgegeben ist, erfolgt die Übernahme von Begriffen und Gedanken oft durch eine entsprechende Anpassung, ja Umdeutung derselben. Letzteres geht deutlich aus meinen Ausführungen hinsichtlich der Bezeichnung des Menschen als politisches Lebewesen bei Basilios hervor.

\section{ANMERKUNGEN}

* Der vorliegende Text ist eine Mitteilung, die beim Regensburger Ökumenischen Symposion 1979 „Die Heiligen der einen Kirche am Beispiel des H1. Basilius (†379)“ gehalten wurde. 
1 Aristoteles, Polit. I, 3; 1253 a 2. Eth. Nic. I, 5; 1097 b 11. An beiden Stellen bezieht sich der Ausdruck auf das Autarkie-Motiv.

2 Vgl. Stobaios, Ecl. II, 59,4: SVF, III, 63,250 Marcianus, Instit. I,1: SVF, III, 77,38. G. Gemistos Plethon, Nomoi, 3,31: Alexandre, S. 124; s. hierzu Th. Nikolaou,

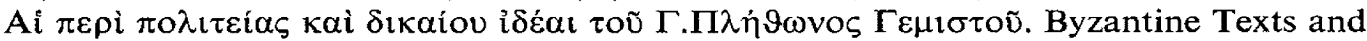
Studies, 13 (Thessaloniki 1974) $56 \mathrm{f}$.

3 Näher auf diesen Vers geht Basilios in der zweiten Homilie zum 14. Psalm ein, die

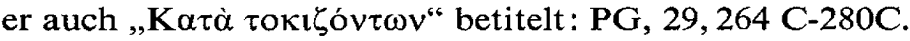

4 Basilios kennt selbstverständlich auch diese Bedeutung; vgl. Eunomii liber apol., 24: PG, 30,861 B; In Psalm. 45, 4: PG, 29, 421 D.

5 Vgl. Plutarch, Über Monarchie und Demokratie und Oligarchie, 2-3 (826 CD).

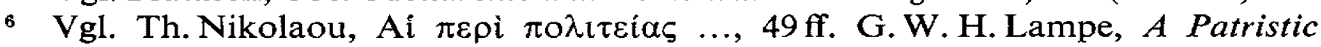

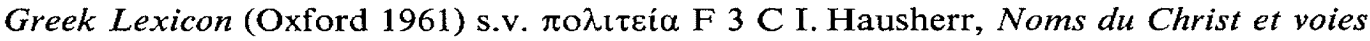
d'oraison. Orientalia Christiana Analecta, 157 (Roma 1960) $164 \mathrm{ff}$.

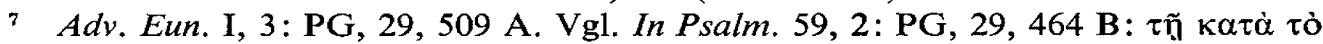

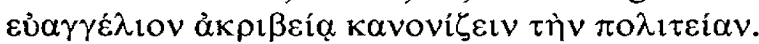

8 In Psalm. 1,4: PG, 29, 220A. Ep. 2,3: PG, 32, 228C. In sanctum baptisma, $7:$ PG, 31, 440A.

9 Vgl. In sanctum baptisma, 7: PG, 31, 440A. In Psalm. 1,2: PG, 29, 213A. In Psalm. 7,3: PG, 29, 236A. De grat. actione, 2; PG, 31, 220D. Ep. 223,2: PG, 32,824C. 10 In Psalm. 59,4: PG, 29, 468B.

11 In Psalm. 45,4-5: PG, 29, 421 B-424 C. Die Kirche als „Polis Gottes“, vgl. auch Klemens A1., Stromata, 4,26: GCS (Stählin), II, 325,1. Origenes, Hom. in Jer.9,2: GCS (Klostermann) 65,21. Athanasios, Exp. in Psalm.71,16: PG, 27, 325 C; Siehe G. Larentzakis, Einheit der Menschheit Einheit der Kirche bei Athanasius. Grazer Theol. Studien, 1 (Graz 1978) 63: „Die Kirche als Stadt ist ... ein bekanntes Symbol, das in der Vätertheologie sehr oft vorkommt".

12 Vgl, auch Klemens A., Stromata, 4, 26: GCS (Stählin) II, 324, 25-28: $\lambda \dot{\varepsilon} \gamma o v \sigma \iota$

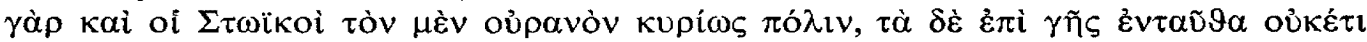

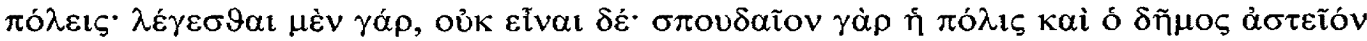

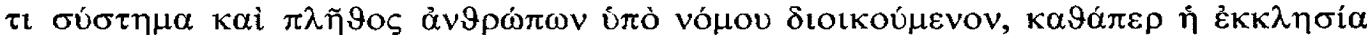

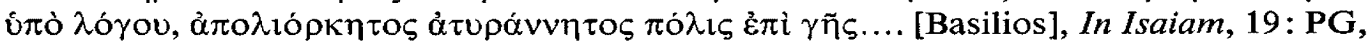

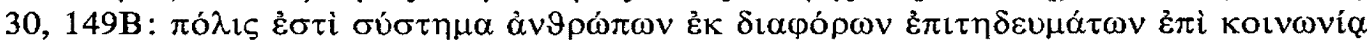

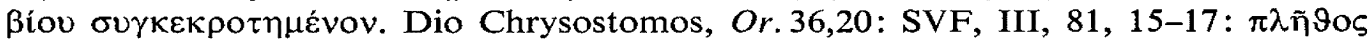

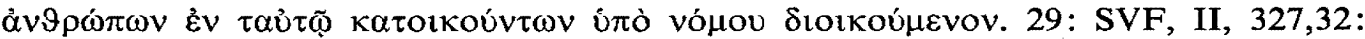

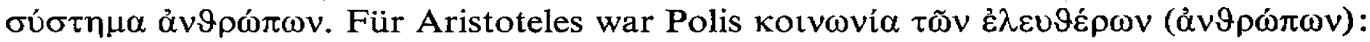
Polit. III,6; 1279 a 21.

13 In sanctum baptisma, 7: PG, 31, 440A. Die übliche Übersetzung von $\pi \circ \lambda i ́ \tau \varepsilon \cup \mu \alpha$ (Phil 3,20) ins Deutsche mit „Heimat" oder „Staat" scheint mir unzutreffend.

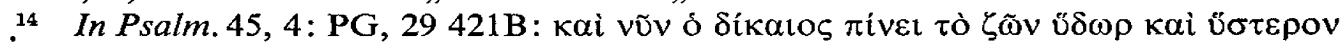

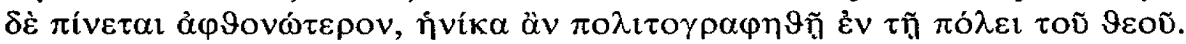

15 Regulae fusius tractatae, interrog. 3,1: PG, 31,917A. Vgl. auch O. Papadopoulou-

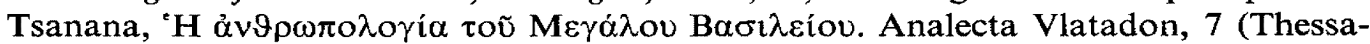

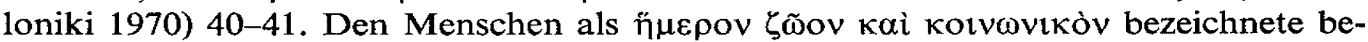
reits Epiktet, Dissertationes, II, 10,14: Schenkl, 147, 5-6; IV, 5,17: Schenk1, 405, 16-17:

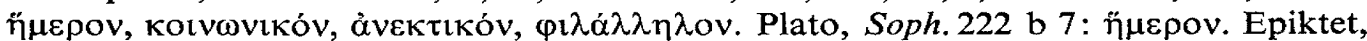

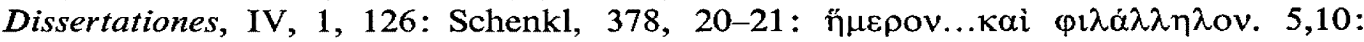

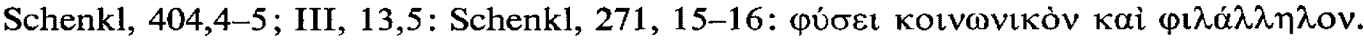

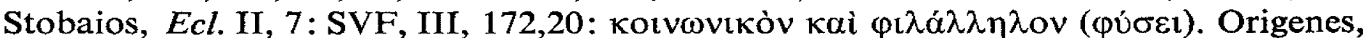
Contra Celsum VIII, so: SVF, III, 85,4. Siehe hierzu auch M. Pohlenz, Die Stoa. Geschichte einer geistigen Bewegung (Göttingen ${ }^{4} 1972$ ) $66 \mathrm{f}$ und 166.

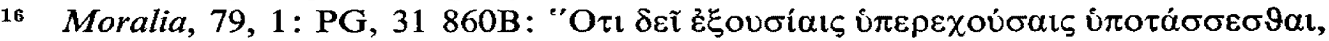




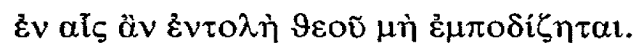

17 In Psalm. 32,9: PG, 29, 344C.

18 O. Gigon, Die antike Kultur and das Christentum (Darmstadt $\left.{ }^{2} 1969\right) 32$.

19 Regulae fusius tractatae, interrog. 3,1: PG, 31, 916D-917A.

20 Polit. I, 3; 1253 a 7-10.

21 J. Hirschberger, Geschichte der Philosophie, I. Altertum und Mittelalter (BaselFreiburg-Wien ${ }^{8}$ 1965) 235.

D-5202 Hennef 1, Mecklenburgerstrasse 1 